\title{
Modal Characteristics and Bistability in Twin Microdisk Photonic Molecule Lasers
}

\author{
Satoru Ishii, Atsuo Nakagawa, and Toshihiko Baba, Member, IEEE
}

\begin{abstract}
We demonstrate coupled-mode characteristics and bistability in photonic molecule lasers composed of evanescentcoupled GaInAsP twin microdisks. First, we show roomtemperature continuous-wave operation by photopumping and discuss the unique behavior of coupled modes, i.e., the anticrossing and splitting characteristics of bonding and antibonding modes. Next, we present the clear bistability, which is observed for the antibonding mode by nonuniform pumping with an effective power of nearly $40 \mu \mathrm{W}$. It is explained by rate equation analysis, which assumes saturable absorption. The analysis also predicts mode switching by the carrier-induced refractive index change. A micron-sized device with a very low power consumption will allow large scale integration of optical memories, optical flip-flops, and so forth.
\end{abstract}

Index Terms-Bistability, microdisk, microlaser, photonic molecule, photonic integration, semiconductor laser.

\section{INTRODUCTION}

$\mathbf{T}$ HE microdisk laser (MDL) [1]-[7] is a microlaser that strongly confines the whispering gallery mode (WGM) by the large refractive index contrast at semiconductor/air boundaries and realizes an ultrasmall cavity [3], [8], [9], an ultralow threshold [9], [10], a high-speed carrier relaxation by the Purcell effect [9], and photonic integration with other elements [11]-[13]. However, the strong optical confinement makes light extraction very difficult. We rather expect some functionality in a photonic molecule structure composed of two or more adjacent MDLs, which are coupled by evanescent waves. The photonic molecule is an optical analog to the chemical molecule. For example, coupled-cavity modes, whose behavior is similar to electronic energy states in a hydrogen molecule, are observed in two coupled vertical-cavity surface-emitting lasers [14] and in coupled microspheres [15]. The photonic molecule of MDLs is advantageous because various molecules can be easily formed by the lithographic technique. In a previous paper, we demonstrated the coupled modes in various types of MDL-based photonic molecules [16].

In this study, we first reconfirmed the low threshold lasing and coupled modes in the photonic molecule of twin MDLs by room-temperature continuous-wave (CW) photopumping. Next, we investigated bistability as a function arising from the coupling between MDLs. In many bistable semiconductor lasers,

Manuscript received December 23, 2004; revised September 29, 2005. This work was supported in part by the IT Program and the 21 st Century COE Program of MEXT and in part by a Grant-In-Aid from the Japan Society for the Promotion of Science.

The authors are with the Department of Electrical and Computer Engineering, Yokohama National University, Yokohama 240-8501, Japan (e-mail: baba@ ynu.ac.jp).

Digital Object Identifier 10.1109/JSTQE.2005.862951 the mechanism of bistability is saturable absorption [17]-[19]. In coupling cavity lasers such as the cleaved coupled-cavity $\left(C^{3}\right)$ laser, the switching occurs between two coupled modes due to gain saturation [20]. These lasers have been expected to be a functional device such as an optical memory and optical flip-flop. However, the conventional devices have a large size of several $100 \mu \mathrm{m}$ in length and consume an operation power of higher than several $10 \mathrm{~mW}$. It is difficult to use them for large-scale photonic integration and sophisticated optical signal processing. In addition, the response time (particularly the fall time) is restricted by the carrier relaxation time, which is of nanosecond order. Recently, another approach is being studied for a similar purpose, i.e., bistable switching in passive microcavities using nonlinear effects such as the Kerr effect [21], [22]. However, the power consumption is still high and/or the available operating bandwidth is restricted by ultrahigh $Q$ factors. Compared with these methods, the MDL photonic molecule has possibilities of large-scale integration, low power consumption, and high-speed operation. Therefore, the investigation of bistability must be an important study for this device.

In Section II, we first show the fabrication process and the room-temperature $\mathrm{CW}$ lasing of this device, which is a fundamental requirement for the bistable operation. Next, in Section III, we discuss coupled-mode characteristics observed in the lasing spectrum with some theoretical results by the finite-difference time-domain (FDTD) method. In Section IV, we describe the rate equation analysis of the bistability based on saturable absorption and the carrier-induced refractive index change. Finally, in Section V, we show its experimental results and discuss the correspondence with theory.

\section{FABRICATION AND LASING CHARACTERISTICS}

The fabrication process is fundamentally the same as that in [16]. However, more details are shown below. The standard laser diode wafer, including five 1.58-GaInAsP quantum wells with a $1 \%$ compressive strain, is used as a substrate. The total thickness of the active layer including the quantum wells and gradientindex separate confinement layers is $240 \mathrm{~nm}$. The active layer is sandwiched by InP layers. On the top of the InP layer, Ti film is evaporated and a resist pattern is formed by e-beam lithography, as shown in Fig. 1(a). Here, two central circles whose diameter $D$ is $2.5-3.0 \mu \mathrm{m}$ will turn into MDLs. The spacing $s$ at the adjacent point between the circles is designed to be $0.2-0.4 \mu \mathrm{m}$. Moreover, smaller circles are drawn as dummy patterns around the central circles. They help to improve the final uniformity of the MDLs. Then, a Cr mask is formed by the evaporation and lift-off technique. The pattern is transferred into Ti by $\mathrm{CF}_{4} / \mathrm{Xe}$ inductive coupling plasma (ICP) etching. Then, 


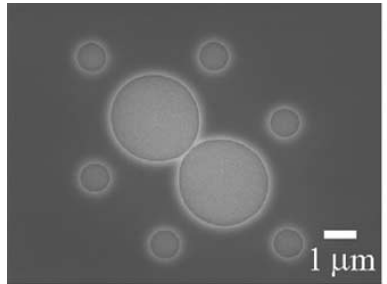

(a)

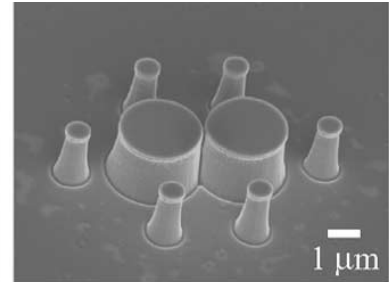

(b)

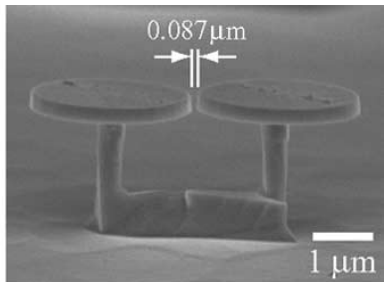

(c)

Fig. 1. Scanning electron microscope images of (a) resist pattern, (b) mesas, and (c) photonic molecule.

mesas, as shown in Fig. 1(b), are formed by $\mathrm{Cl}_{2} / \mathrm{Xe}$ ICP etching. Corresponding to the pattern of Fig. 1(a), dummy mesas are surrounding the central mesas. Finally, the photonic molecule of MDLs is formed by $\mathrm{HCl}$ selective wet etching of InP layers, as shown in Fig. 1(c). The dummy mesas disappear due to the excess wet etching. But they cause InP pedestals of MDLs to be almost at the center. (Without dummy mesas, fast etching from outside makes pedestals close to the adjacent point) The pedestal of each MDL should be narrower than $1.5 \mu \mathrm{m}$ so that the scattering loss of the WGM at the pedestal is negligible [6]. It was nearly $0.5 \mu \mathrm{m}$ in the device of Fig. 1(c). The sidewall roughness of the MDLs was evaluated to be less $10 \mathrm{~nm}$ by a highresolution scanning electron microscope, and the ellipticity was less than $\pm 3 \%$. The spacing $s$ at the adjacent point becomes less than $0.1 \mu \mathrm{m}$ due to the proximity effect in the e-beam lithography and a small tilt in the sidewall of less than $5^{\circ}$. This distance is small enough for the evanescent coupling between MDLs [11].

In the measurement, 980-nm CW laser light is used as a pump source. The multimode-fiber output is focused to a spot diameter of $20 \mu \mathrm{m}$ by a couple of lenses and is irradiated on the sample stage from the top. Due to the multimode-fiber output, the power profile of the pump light is almost flat except at the edge of the spot. The pump light position is controlled by the piezo stage at the fiber connector with a spatial resolution on the sample stage of $20 \mathrm{~nm}$. The temperature of the sample stage can be controlled by a Peltier cooler. In the measurement of normal lasing characteristics, a device is put at the center of the light spot. The light output from the device is detected by a tapered multimode fiber whose tip is placed close to the device from the lateral side and analyzed by an optical spectrum analyzer. The pump source, piezo stage, and optical spectrum analyzer are controlled by computer.

An example of room-temperature lasing characteristics for $D=2.6 \mu \mathrm{m}$ is shown in Fig. 2. Here, the upper lateral axis of Fig. 2(a) shows the irradiated power to the device $P_{\text {irr }}(3.4 \%$ of total power irradiated on the sample stage), which is estimated by considering the ratio of the total area of two MDLs to the focused spot area. The lower lateral axis denotes the effective pump power $P_{\text {eff }}$ (33\% of $P_{\text {irr }}$ ), which is estimated by considering the resonant absorption [23] with an average absorption coefficient of $20000 \mathrm{~cm}^{-1}$ of the MDL, an MDL thickness of 240 $\mathrm{nm}$, and a reflectivity at MDL surfaces of 30\%. The threshold is estimated to be $65 \mu \mathrm{W}$. For many samples, it ranged from 65 to $152 \mu \mathrm{W}$. This value is slightly higher than the minimum value obtained previously [16]. It might be caused by the different sidewall roughness and different distances of the adjacent point.

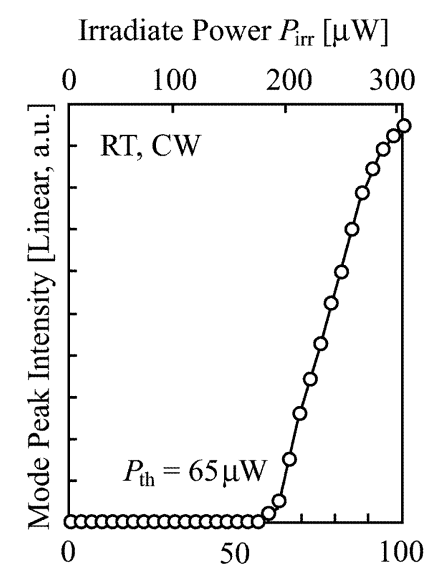

Effective Pump Power $P_{\text {eff }}[\mu \mathrm{W}]$

(a)

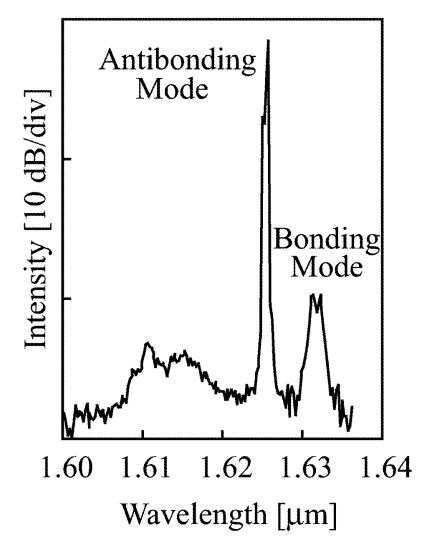

(b)
Fig. 2. Measured lasing characteristics of photonic molecule by uniform $\mathrm{CW}$ photopumping at room temperature. (a) Light intensity characteristic. (b) Lasing spectrum at nearly 1.5 times the threshold.

\section{OPTICAL COUPLing}

In the spectrum shown in Fig. 2(b), two strong peaks are observed, and each top separates into two with a spacing of $\sim 1 \mathrm{~nm}$. Moreover, a very slow and weak peak appears at the shorter wavelength side. These peaks are observed repeatedly for many samples. To know their origins, we analyzed the coupled modes by the FDTD method. We omit to explain the detail of the FDTD method, since it is a standard mode calculation method that is widely used for microcavity studies. The detail is almost the same as that in [11]. First, two MDLs with $D=3.0 \mu \mathrm{m}$ and $s=0.1 \mu \mathrm{m}$ are assumed. An in-plane polarized wave is excited in one MDL in order to simulate the polarized emission in compressively strained quantum wells [6]. The magnetic field distribution in the vertical direction $H_{z}$ and resonant spectra are calculated by the fine tuning of the excited wavelength and position. Fig. 3 shows two types of modes, i.e., the bonding mode which couples by the in-phase at the adjacent point and appears at the longer wavelength side, and the antibonding mode which couples by the out-of-phase and appears at the shorter wavelength side. For each mode, there are two phase states 0 and $\pi$ (they are 0 and $\pi$ when considering the phase of the standing wave) at the adjacent point. In general, the antibonding mode has a higher $Q$ factor. This is considered to be due to the large penetration of the modal electric field into the interdisk spacing for the assumed polarization and a smaller scattering loss for 


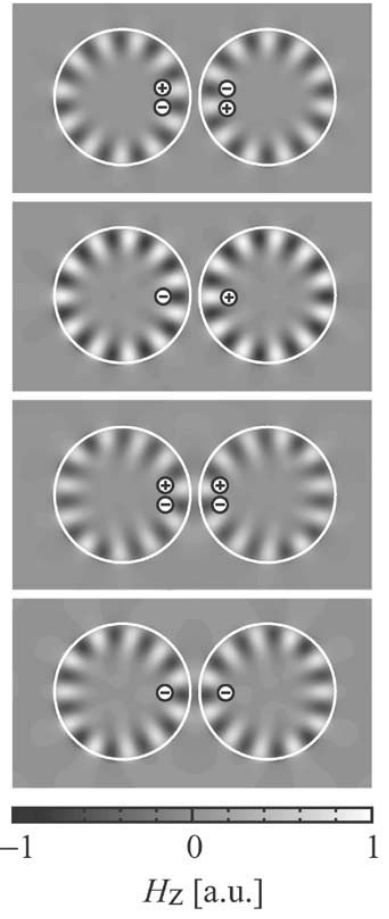

(a)

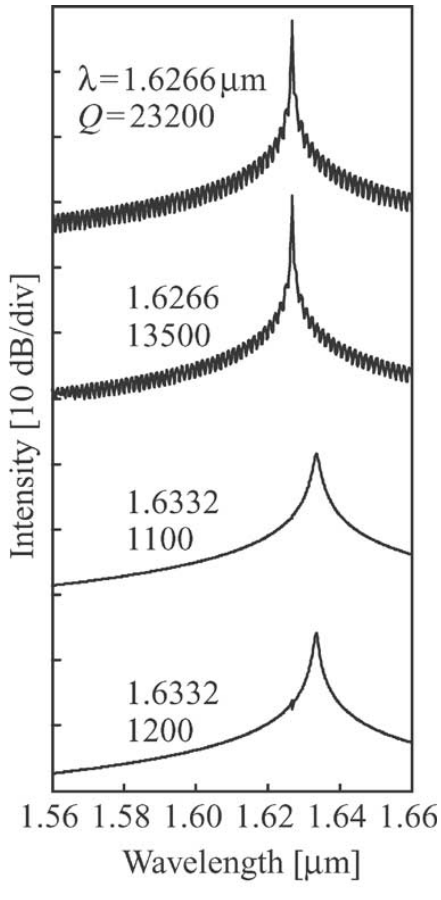

(b)
Fig. 3. Modal characteristics of photonic molecule calculated by FDTD method. (a) Magnetic field profiles $H_{z}$ of each mode. Plus and minus symbols denote signs of the field at the adjacent point between MDLs. (b) Resonant spectra. Lower two are bonding modes and upper two are antibonding modes.

the antibonding mode having a node at the center of the interdisk spacing. As seen in the spectra, different phase states have the same wavelength, so they are degenerate. Therefore, only two resonant wavelengths are observed ideally. However, in the real device, the degeneracy can be broken by the ellipticity and small disordering coming from the roughness and proximity effect. The separation of each peak in the spectrum of Fig. 2(b) is caused by such broken degeneracy. Although the detail is not shown here, the slow peak at the shorter wavelength side can be explained by a higher order WGM rounding inside of the MDL and having a lower $Q$. In the following, we ignore this mode because it is outside the interest of this study.

The strength of the coupling depends on the difference between the original resonant wavelengths of the MDLs. It is nearly proportional to the difference in diameter between MDLs. Fig. 4 shows the modal wavelength shift versus the diameter difference $\Delta D=D_{1}-D_{2}$, when the diameter of one MDL (MDL1) is fixed and that of the other MDL (MDL2) is changed. For the experimental plots, some devices having a relatively small $\Delta D$ and $s \sim 0.2 \mu \mathrm{m}$ were selected, and each $\Delta D$ was obtained by measuring diameters of MDLs (average value of $x$ and $y$ diameters). Theoretical curves and corresponding field profiles are also shown. The fixed wavelength mode (almost horizontal solid line) arises from MDL1 and the shifted wavelength mode (oblique dashed line) arises from MDL2. When these wavelengths are distant from each other, the coupling is weak, so the intensity of each mode is localized in either MDL.

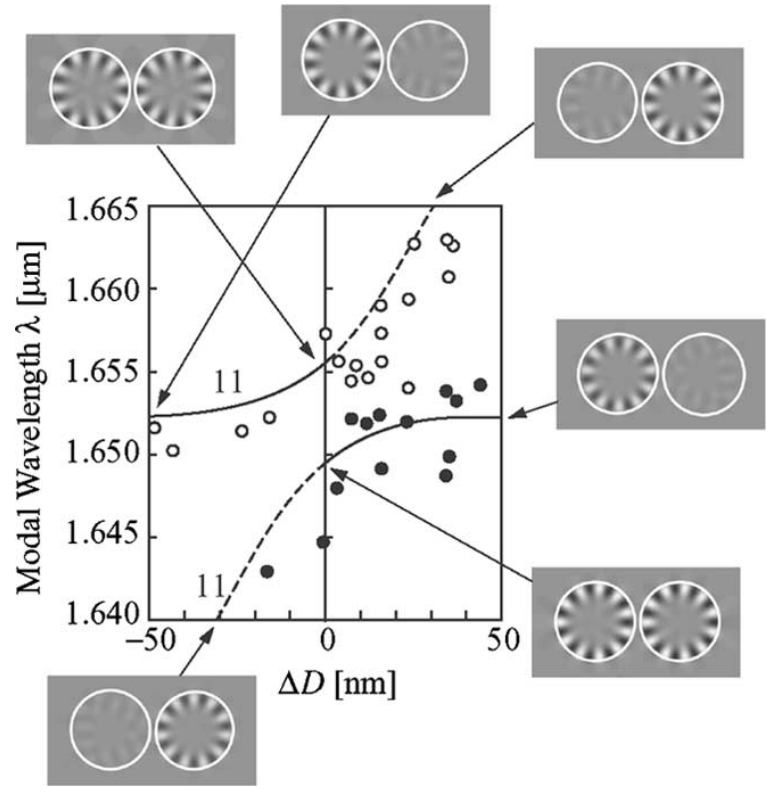

Fig. 4. Modal wavelength characteristic for diameter detuning between two MDLs $\Delta D$. Open and closed circles denote experimental plots for bonding and antibonding modes, respectively. Solid and dashed lines originate from MDL1 and MDL2, respectively. Corresponding field profiles $H_{z}$ calculated by the FDTD method are also shown.

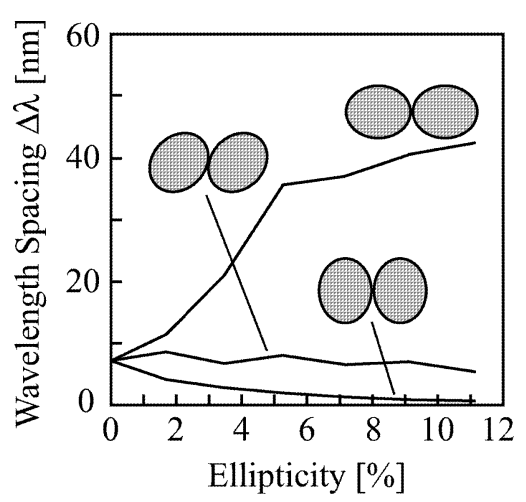

Fig. 5. Wavelength spacing calculated with ellipticity of MDLs. Three different orientations of elliptical MDLs against the adjacent point are considered.

When the wavelengths are close to each other, the anticrossing characteristic appears, which indicates efficient coupling. On this condition, two separate curves correspond to the bonding and antibonding modes, whose field intensities are equal between MDLs. Experimental plots agree well with the expectation. Such a characteristic should not appear when each MDL resonates individually. The data spread of less than $5 \mathrm{~nm}$ in wavelength is thought to be caused by the ellipticity of the MDLs. Fig. 5 shows the wavelength spacing of the bonding and antibonding modes $\Delta \lambda$ calculated as a function of the ellipticity for two MDLs with $D=3.0 \mu \mathrm{m}$ for the long axis and $s=0.1 \mu \mathrm{m}$. When the adjacent point is directed toward the long or short axis, two modes shift with increasing or decreasing $\Delta \lambda$, respectively, as the ellipticity increases. This is because the edge of the long axis has a smaller bending radius, which increases 


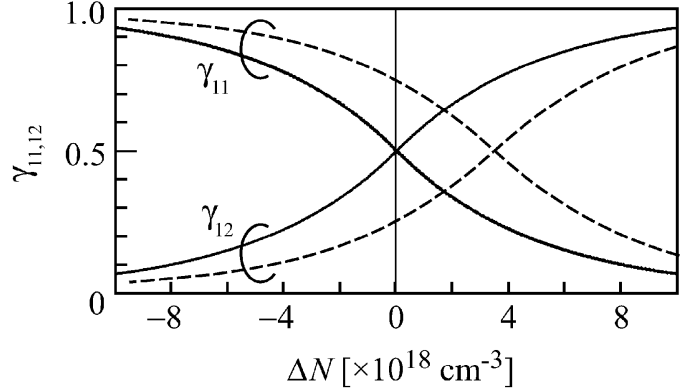

Fig. 6. Mode localization factors $\gamma_{11}$ and $\gamma_{12}$ calculated with carrier density difference. The FDTD method is used with consideration of the carrier plasma effect. Solid and dashed lines are obtained for $\Delta D=0 \mathrm{~nm}$ and $20 \mathrm{~nm}$, respectively.

the penetration depth of the evanescent field of the mode and enhances the coupling strength for the constant $s$. When the adjacent point is directed in the middle of the long and short axes, $\Delta \lambda$ is kept almost constant. In the experiment, $\pm 3 \%$ ellipticity was observed, as mentioned previously. It was noticed that the long axis of the ellipse was parallel to the direction of e-beam scanning. Therefore, we employed the e-beam toward the middle of the long and short axes to minimize the influence. Still, a change of several $\mathrm{nm}$ in $\Delta \lambda$ is unavoidable. The data spread in Fig. 4 can be explained by this effect.

As will be discussed in Section IV, the carrier density difference $\Delta N$ is induced between MDLs by nonuniform pumping. In general, the carrier plasma effect changes the modal index $n_{m}$ by $\Delta n_{m}=-e^{2} \Gamma \Delta N / 8 \pi^{2} \mathrm{~nm}^{*} \varepsilon_{0} \nu^{2}$, where $\Gamma$ is the optical confinement factor in the MDL, $e$ the electron charge, $n$ the material index, $m^{*}$ the effective mass of electrons $\left(=0.049 m_{0}\right), \varepsilon_{0}$ the vacuum dielectric constant, and $\nu$ the frequency of light (189 THz for $\lambda=1.584 \mu \mathrm{m}$ ). Similarly to the case with the diameter difference $\Delta D, \Delta n_{m}$ changes the coupling condition and localizes the mode field into either MDL. Let us define the mode localization factors $\gamma_{11}$ and $\gamma_{12}$ as ratios of the field energy of the bonding and antibonding modes localized in MDL1, respectively, and $\gamma_{1}$ and $\gamma_{22}$ as those localized in MDL2, respectively. Obviously, $\gamma_{11} \gamma_{21}=1, \gamma_{12} \gamma_{22}=1$. Solid lines in Fig. 6 show $\gamma_{11}$ and $\gamma_{12}$ calculated for $\Delta N=N_{1}-N_{2}$ and $\Delta D=0 \mu \mathrm{m}$. It is easily understood that $\gamma_{11}=\gamma_{12}=0.5$ when $\Delta N=0 \mathrm{~cm}^{-3}$. If $\Delta N$ is increased by decreasing $N_{2}, \gamma_{11}$ decreases and $\gamma_{12}$ increases. This means that the bonding and antibonding modes are localized into MDL2 and MDL1, respectively. Therefore, nonuniform pumping simply weakens the coupling. If a positive $\Delta D$ is given intentionally, the mode localization is reduced and the coupling is strengthened by $\Delta N$, as indicated by dashed lines in Fig. 6 .

\section{RATE EQUATION ANALYSIS}

This section theoretically discusses bistability in the presence of nonuniform pumping. For carrier densities of MDL1 and MDL2, $N_{1}$ and $N_{2}$, respectively, and photon densities of the bonding and antibonding modes, $S_{1}$ and $S_{2}$, respectively, rate

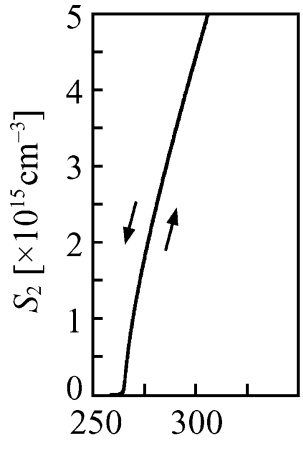

(a)

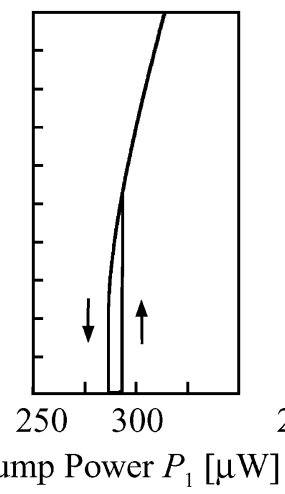

(b)

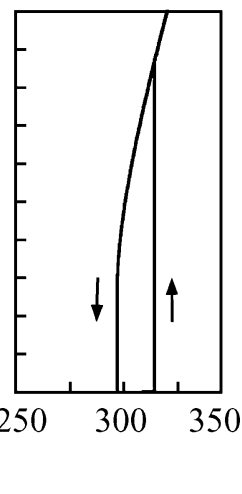

(c)
Fig. 7. Lasing characteristics of $S_{2}$ by rate equation analysis, assuming fixed mode localization factors of 0.5 . Pumping ratio $P_{2} / P_{1}$ is (a) 0.2 , (b) 0.1 , and (c) 0 .

equations are expressed as

$$
\begin{aligned}
\frac{d N_{1}}{d t}= & P_{1}-\frac{N_{1}}{\tau_{c}\left(N_{1}\right)}-\Gamma G\left(N_{1}\right) \cdot\left(\gamma_{11} S_{1}+\gamma_{12} S_{2}\right) \\
\frac{d N_{2}}{d t}= & P_{2}-\frac{N_{2}}{\tau_{c}\left(N_{2}\right)}-\Gamma G\left(N_{2}\right) \cdot\left(\gamma_{21} S_{1}+\gamma_{22} S_{2}\right) \\
\frac{d S_{1}}{d t}= & \operatorname{CB}\left\{\gamma_{11} N_{1}^{2}+\gamma_{21} N_{2}^{2}\right\} \\
& +\Gamma\left\{\gamma_{11} G\left(N_{1}\right)+\gamma_{21} G\left(N_{2}\right)\right\} S_{1}-\frac{S_{1}}{\tau_{1}} \\
\frac{d S_{2}}{d t}= & \operatorname{CB}\left\{\gamma_{12} N_{1}^{2}+\gamma_{22} N_{2}^{2}\right\} \\
& +\Gamma\left\{\gamma_{12} G\left(N_{1}\right)+\gamma_{22} G\left(N_{2}\right)\right\} S_{2}-\frac{S_{2}}{\tau_{2}}
\end{aligned}
$$

where $P_{1}$ and $P_{2}$ are pump rate densities, $\tau_{c}(N)$ the carrier lifetime, $G(N)$ the gain coefficient, $C$ the spontaneous emission factor, $B$ the radiative recombination coefficient, and $\tau_{1}$ and $\tau_{2}$ the photon lifetimes. In calculating $N_{1}, N_{2}, S_{1}$, and $S_{2}$ by solving (1)-(4), some typical material parameters for GaInAsP were used, i.e., $\Gamma=0.78, C=0.0001, B=3.3 \times 10^{-10} \mathrm{~cm} / \mathrm{s}$. To simulate the compressively strained quantum wells, the logarithmic gain expressed as $G(N)=\left(c / n_{g}\right) g \log \left(N / N_{0}\right)$ is used with the vacuum velocity of light $c$, a group index $n_{g}$ of 3.5 , a gain coefficient $g$ of $1500 \mathrm{~cm}^{-1}$, and a transparent carrier density $N_{0}$ of $1.5 \times 10^{18} \mathrm{~cm}^{-3}$. The carrier lifetime $\tau_{c}(N)$ is given by $(A+\mathrm{BN})^{-1}$, where $A=1.0 \times 10^{8} \mathrm{~s}^{-1}$ is the nonradiative recombination coefficient mainly determined by surface recombination at the sidewall of the MDLs. In addition, a scattering loss of modes by the sidewall roughness $\alpha_{s}$ of $20 \mathrm{~cm}^{-1}$, a free carrier absorption loss in the quantum wells of $25 \mathrm{~cm}^{-1}$, and $Q_{1}=20000$ and $Q_{2}=1000$ are assumed. Then, $\tau_{1}$ and $\tau_{2}$ are calculated to be $2.7 \mathrm{ps}$ and $1.4 \mathrm{ps}$, respectively.

Let us first assume $\gamma_{11}=\gamma_{12}=\gamma_{21}=\gamma_{22}=0.5$ for simplicity, and $D_{1}=D_{2}=3.0 \mu \mathrm{m}$. Then, static lasing characteristics are calculated, as shown in Fig. 7. When the strongly nonuniform pumping of $P_{2} / P_{1}<0.1$ is assumed, the bistability due to 

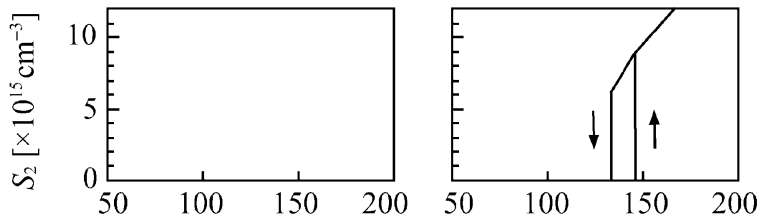

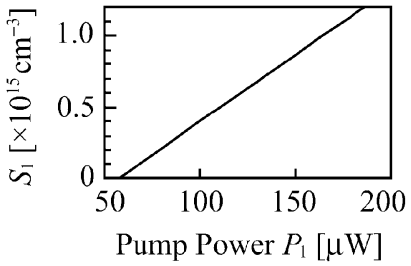

(a)

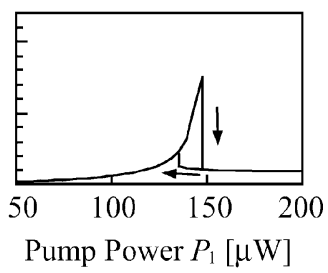

(b)
Fig. 8. Lasing characteristics of $S_{1}$ (lower) and $S_{2}$ (upper) by rate equation analysis, assuming variable mode localization factors. (a) $\Delta D=0 \mathrm{~nm}$. (b) $\Delta D=20 \mathrm{~nm}$.

the saturable absorption appears only in $S_{2}$ with the higher $Q$ factor. When $P_{2} / P_{1}$ is 0.2 , the threshold is reduced, while the bistability disappears. Actually, however, $\gamma_{11}, \gamma_{12}, \gamma_{21}$, and $\gamma_{22}$ can be changed by $N_{1}$ and $N_{2}$, as mentioned previously. When $\gamma_{11}$ and $\gamma_{12}$, shown by solid lines in Fig. 6, are used, the lasing characteristic for $P_{2} / P_{1}=0$ is calculated as shown in Fig. 8(a). When $\Delta N$ increases against nonuniform pumping, $\gamma_{12}$ becomes dominant. This weakens the mode coupling and reduces saturable absorption. This is the reason that Fig. 8(a) does not show bistability but rather the normal lasing in $S_{2}$. Next, let us use dashed lines in Fig. 6 for $\gamma_{11}$ and $\gamma_{12}$ to take account of different MDL diameters. Then, the dominant factor changes from $\gamma_{11}$ to $\gamma_{12}$ as $\Delta N$ increases. The lasing characteristic calculated for $D_{1}=3.02 \mu \mathrm{m}$ and $D_{2}=3.00 \mu \mathrm{m}$ is shown in Fig. 8(b). Here, both $S_{1}$ and $S_{2}$ show bistability, and mode switching is observed; $S_{2}$ starts lasing for a lower pump level with a weaker intensity, but the lasing switches to $S_{1}$ with a stronger intensity.

\section{EXPERIMENT}

The nonuniform pumping was carried out using the measurement setup described in Section II. Here, the pump intensity was raised up and sent down step by step, and two peak wavelengths and intensities of the coupled modes were recorded. First, the device was uniformly pumped near the center of the pump spot. Next, the pump spot was moved so that its edge finally touched the device. On this condition, the ratio of the nonuniform pumping $P_{2} / P_{1}$ was roughly estimated from the observation of a GaInAs infrared camera. Even though each MDL is nonuniformly pumped, the carrier distribution in each MDL will be uniform due to the carrier diffusion. Therefore, $\Delta N$ as described in Section IV is realized by this method. In order to reduce the thermal effect, the temperature of the sample stage was decreased to $-5{ }^{\circ} \mathrm{C}$. Two kinds of measured results for the antibonding mode at the short wavelength side are shown in Fig. 9. Here, the lateral axis denotes the effective pump power estimated similarly to the case of Fig. 2. One different point is the estimation of $P_{\text {irr. }}$. For this, we took account of the actually irradiated area in two MDLs under a nonuniform pumping con-

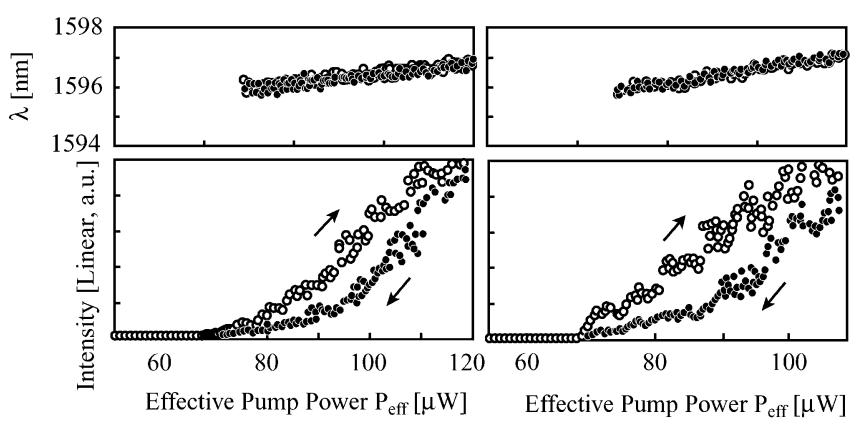

(a)

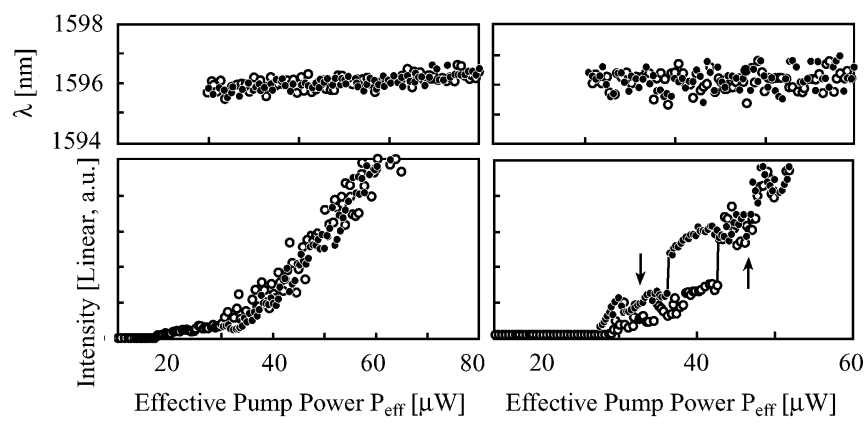

(b)

Fig. 9. Light intensity and wavelength characteristics measured in the experiment. Open and closed circles are obtained for raising up and sending down the pump power, respectively. Left is the uniform pumping and right the nonuniform pumping with $P_{2} / P_{1} \sim 0.2$. (a) High threshold device. (b) Low threshold device.

dition. The total time for measuring one characteristic is over 20 min. Fig. 9(a) shows the lasing characteristic with slow hysteresis. In this case, the threshold is as high as $\sim 80 \mu \mathrm{W}$. Hysteresis showed the clockwise loop, and this characteristic is almost independent of the uniformity or nonuniformity of pumping. The lasing wavelength shows the redshift even below threshold. Therefore, it is reasonable to consider that hysteresis was caused by the heat during measurement. On the other hand, Fig. 9(b) does not show hysteresis but rather a simple lasing characteristic with a low threshold of $30 \mu \mathrm{W}$ for uniform pumping. Even for the nonuniform pumping of $P_{2} / P_{1}=0.2$, the threshold is of the same order $(45 \mu \mathrm{W}$ and $35 \mu \mathrm{W}$ for increasing and decreasing the pumping) as for the uniform pumping, and the abrupt hysteresis with the anticlockwise loop is observed. In addition, a clear redshift is not seen in the lasing wavelength. Therefore, this bistability is thought to be due to saturable absorption rather than thermal hysteresis. This result was reproducible for some devices.

All of the above results were obtained for the antibonding mode. Most devices exhibited the bonding mode with the much lower intensity, so clear and systematic evaluation was difficult. A limited number of devices showed clear lasing by the bonding mode. However, only the thermal hysteresis similar to Fig. 9(a) was observed, and a clear correlation between the bonding and antibonding modes, which should be evidence of mode switching, was not confirmed. One interesting result was the mode switching against the position shift of the pump light. Fig. 10 shows a series of lasing spectra above threshold when the pump position is shifted toward increasing nonuniformity. First, both 


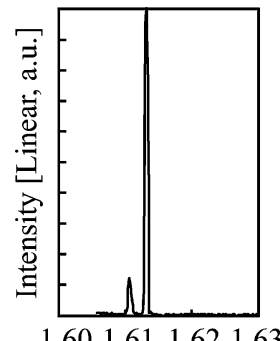

(a)

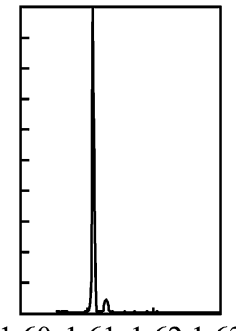

1.601 .611 .621 .63

Wavelength $[\mu \mathrm{m}]$

(b)

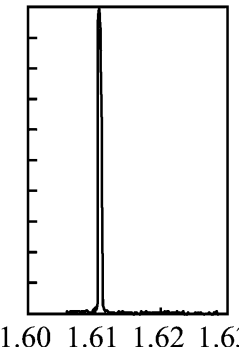

(c)
Fig. 10. Lasing spectra observed for a device with fixed pump power. $P_{2} / P_{1}$ is nearly (a) 1.0 , (b) 0.8 , and (c) 0.6 .

modes are observed, but the bonding mode at the longer wavelength side is more intense. As nonuniformity increases, the intensities of modes exchange and the antibonding mode becomes dominant. This result suggests the behavior of the mode localization factor similar to the solid lines in Fig. 6; the accidental small diameter difference $\Delta D$ in the device first satisfies the lasing condition of both modes, but the carrier density difference $\Delta N$ by nonuniform pumping makes the antibonding mode dominant. To observe the mode switching, as shown in Fig. 8(b), the behavior of the dashed curves in Fig. 6 by the intentionally given $\Delta D$ is necessary.

\section{CONCLUSION}

In conclusion, room-temperature $\mathrm{CW}$ lasing was observed in the photonic molecule of GaInAsP twin MDLs by photopumping. The two mode peaks observed in the lasing spectrum were confirmed to be the bonding and antibonding modes between the MDLs from the anticrossing characteristic against the detuning of MDL diameters. Rate equation analysis predicted simple bistability by saturable absorption and mode switching by the carrier-induced refractive index change. In the experiment, the sharp bistability was observed by nonuniform pumping with an effective pump power of less than $70 \mu \mathrm{W}$. Some observations indicated that it was not a thermal hysteresis but a hysteresis based on saturable absorption. The behavior of modes indicated that mode switching requires the fine control of disk diameters to obtain a situation in which the carrier-induced index change leads the dominant mode switching from the antibonding mode to the bonding mode. The mode switching discussed here is restricted by the carrier relaxation. However, we can consider a more high-speed switching based on cross gain modulation [24].

\section{REFERENCES}

[1] S. L. McCall, A. F. J. Levi, R. E. Slusher, S. L. Pearton, and R. A. Logan, "Whispering gallery mode microdisk lasers," Appl. Phys. Lett., vol. 60, pp. 289-291, 1992.

[2] A. F. J. Levi, R. E. Slusher, S. L. McCall, T. Tanbun-Ek, D. L. Coblentz, and S. J. Pearton, "Room temperature operation of microdisk lasers with submilliamp threshold current," Electron. Lett., vol. 28, pp. 1010-1012, 1992.

[3] A. F. J. Levi, S. L. McCall, S. J. Pearton, and R. A. Logan, "Room temperature operation of submicrometer radius disc laser," Electron. Lett., vol. 29, pp. 1666-1667, 1993.
[4] U. Mohideen, W. S. Hobson, S. J. Pearton, F. Ren, and R. E. Slusher, "GaAs/AlGaAs microdisk lasers," Appl. Phys. Lett., vol. 64, pp. 19111913, 1994.

[5] T. Baba, "Photonic crystals and microdisk cavities based on GaInAsP/InP system," IEEE J. Sel.Topics Quantum Electron., vol. 3, no. 3, pp. 808-830, Jun. 1997.

[6] M. Fujita, A. Sakai, and T. Baba, "Ultra-small and ultra-low threshold microdisk injection laser: Design, fabrication, lasing characteristics, and spontaneous emission factor," IEEE J. Sel. Topics Quantum Electron., vol. 5, no. 3, pp. 673-681, May/Jun. 1999.

[7] D. S. Song, J. K. Hwang, C. K. Kim, I. Y. Han, D. H. Jang, and Y. H. Lee, "InGaAsP Microdisk Lasers on $\mathrm{Al}_{x} \mathrm{O}_{y}$," IEEE Photon. Technol. Lett., vol. 12, no. 8, pp. 954-956, Aug. 2000.

[8] T. Baba, M. Fujita, A. Sakai, M. Kihara, and R. Watanabe, "Lasing characteristics of GaInAsP-InP strained quantum-well microdisk injection lasers with diameter of 2-10 $\mu \mathrm{m}$," IEEE Photon. Technol. Lett., vol. 9, no. 7, pp. 878-880, Jul. 1997.

[9] T. Baba and D. Sano, "Low threshold lasing and Purcell effect in microdisk lasers at room temperature," IEEE J. Sel. Topics Quantum Electron., vol. 9, no. 5, pp. 1340-1346, Sep./Oct. 2003.

[10] M. Fujita, R. Usigome, and T. Baba, "Continuous wave lasing in GaInAsP microdisk laser with threshold of $40 \mu \mathrm{A}$," Electron. Lett., vol. 36, pp. 790791, 2000.

[11] A. Sakai and T. Baba, "FDTD simulation of photonic devices and circuits based on circular and fan-shaped disks," J. Lightwave Technol., vol. 17, no. 8, pp. 1493-1499, Aug. 1999.

[12] D. Y. Chu, M. K. Chin, W. G. Bi, H. Q. Hou, C. W. Tu, and S. T. Ho, "Double-disk structure for output coupling in microdisk lasers," Appl. Phys. Lett., vol. 65, pp. 3167-3169, 1994.

[13] S. J. Choi, Z. Peng, Q. Yang, S. J. Choi, and P. D. Dapkus, "Eightchannel microdisk CW laser arrays vertically coupled to common output bus waveguides," IEEE Photon. Technol.Lett., vol. 16, no. 2, pp. 356-358, Feb. 2004.

[14] M. Bayer, T. Gutbrod, J. P. Reithmaier, and A. Forchel, "Optical mode in photonic molecules," Phys. Rev. Lett., vol. 81, pp. 2582-2585, 1998.

[15] T. Mukaiyama, K. Takeda, H. Miyazaki, Y. Jimba, and M. KuwataGonokami, "Tight-binding photonic molecule modes of resonant bisphere," Phys. Rev. Lett., vol. 82, pp. 4623-4626, 1999.

[16] A. Nakagawa, S. Ishii, and T. Baba, "Photonic molecule laser composed of GaInAsP microdisks," Appl. Phys. Lett., vol. 86, pp. 041-112, 2005.

[17] M. Ueno and R. Lang, "Conditions for self-sustained pulsation and bistability in semiconductor lasers," J. Appl. Phys, vol. 58, pp. 1689-1692, 1985.

[18] Y. Hori, H. Sato, H. Serizawa, and T. Kajiwara, "Effect of device parameters on bistable semiconductor laser," Appl. Phys., vol. 60, no. 2, pp. 534-537, 1986.

[19] H. Kawaguchi, "Optical bistability and chaos in a semiconductor laser with a saturable absorber," Appl. Phys. Lett., vol. 45, pp. 1264-1266, 1984.

[20] G. P. Agrawal and N. K. Dutta, "Optical bistability in coupled-cavity semiconductor lasers," J. Appl. Phys., vol. 56, pp. 664-669, 1985.

[21] D. K. Armani, T. J. Kippenberg, S. M. Spillane, and K. J. Vahala, "Ultrahigh-Q toroid microcavity on a chip," Nature, vol. 424, pp. 925-928, 2003.

[22] V. R. Almeida, C. A. Barrios, R. R. Panepucci, and M. Lipson, "All-optical control of light on a silicon chip," Nature, vol. 431, pp. 1081-1084, 2004

[23] K. Nozaki, A. Nakagawa, D. Sano, and T. Baba, "Ultralow threshold and singlemode lasing in microgear lasers and its fusion with quasiperiodic photonic crystals," IEEE J. Sel. Topics Quantum Electron., vol. 9, no. 5, pp. 1355-1360, Sep./Oct. 2003.

[24] M. T. Hill, H. J. S. Dorren, T. de Vries, X. J. M. Leijtens, J. H. den Besten, B. Smalbrugge, Y-S. Oei, H. Binsma, G-D. Khoe, and M. K. Smit, "A fast low-power optical memory based on coupled micro-ring lasers," Nature, vol. 432, pp. 206-209, 2004.

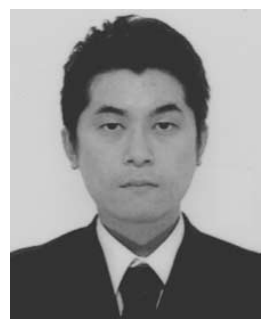

Satoru Ishii was born in Kanagawa, Japan, on September 6, 1981. He received the B.E. degree in electrical and computer engineering from Yokohama National University, Yokohama, Japan, in 2004 where he is currently working toward the M.E. degree.

Mr. Ishii is a member of the Japanese Society of Applied Physics (JSAP). 
Atsuo Nakagawa, photograph and biography not available at the time of publication.

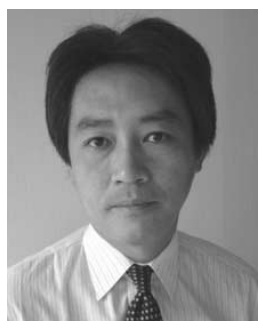

Toshihiko Baba (M'93) received the Ph.D. degree in electrical and computer engineering from Yokohama National University (YNU), Yokohama, Japan, in 1990 .

During his Ph.D. work, he was engaged in ARROW waveguides and photonic lightwave circuits. From 1991 to 1993, he was with the Tokyo Institute of Technology as a Research Associate. He discussed spontaneous emission control in vertical cavity surface emitting lasers (VCSELs) and achieved roomtemperature continuous-wave operation in a longwavelength VCSEL. In 1994, he became an Associate Professor at YNU and started research on photonic crystals (PCs). He first demonstrated InP-based light emitters, line defect waveguides, the smallest nanolaser, the strong Purcell effect, a high-efficiency LED, a high-power holey VCSEL, and so on in PCs. He also proposed an optical buffer utilizing slow light in the PC waveguide and a compact demultiplexer utilizing superprism optics. Also, he is active in microdisk lasers and Si photonic wire waveguides. He achieved the record low threshold in GaInAsP injection lasers by microdisk and demonstrated an ultracompact arrayed waveguide grating by photonic wire waveguide. In 2005, he became a Full Professor at YNU.

Dr. Baba is a member of the Institute of Electronics, Information, and Communication Engineers (IEICE), the Japan Society of Applied Physics, the Optical Society of America, and the American Physics Society. He received the Niwa Memorial Prize in 1991, the Best Paper Award of the Micro-Optic Conference in 1993 and 1999, the Best Paper Award and Academic Encouragement Award from the IEICE in 1994, and the Marubun Research Encouragement Award in 2000 . 